\title{
General methods for free-volume theory
}

\author{
S. M. Oversteegen \\ Van't Hoff-Laboratorium voor Fysische en Collö̈dchemie, Debye-Instituut, Universiteit Utrecht, Padualaan \\ 8, $3584 \mathrm{CH}$ Utrecht, The Netherlands \\ R. Roth \\ Max-Planck-Institut für Metallforschung, Heisenbergstrasse 3, D-70569 Stuttgart, Germany, \\ and Institut für Theoretische und Angewandte Physik, Universität Stuttgart, Pfaffenwaldring 57, \\ D-70569 Stuttgart, Germany
}

(Received 12 January 2005; accepted 21 March 2005; published online 1 June 2005)

\begin{abstract}
Free-volume theory for understanding depletion phenomena in mixtures of two species is generally derived using scaled-particle theory for those specific entities. Here we first give a general scaled-particle method for convex bodies in terms of the characteristic geometrical measures of the depletion agent, i.e., its volume, surface area, and integrated mean curvature, in mixtures with hard spheres. Second, we show that similar results can be derived from fundamental-measure theory. This different approach allows us to get a deep insight into the meaning of the various contributions to the theory from a geometrical point of view. From these two methods we arrive at a generalized "recipe" to free-volume theory. This recipe can be based on a desired equation of state for any convex shape of the depletion agents and is also valid for (polydisperse) mixtures of those. This is illustrated by mixtures of spheres with ellipsoids, spheres with several geometries as models for disklike mesogens, e.g., gibbsite, as well as depletion of spheres due to bar-shaped colloids, e.g., goethite. (C) 2005 American Institute of Physics. [DOI: 10.1063/1.1908765]
\end{abstract}

\section{INTRODUCTION}

Mixtures of colloidal particles with different sizes and shapes are ubiquitous in industry, food science, and the biological realm. ${ }^{1,2}$ Moreover, there is much interest in understanding the properties of mixed colloidal suspensions at a fundamental microscopic level. It is commonly accepted that mutual asymmetry of the particles in these mixtures alone may induce a net attraction between them by the so-called depletion effect. ${ }^{3-6}$

For binary mixtures of asymmetric hard spheres this depletion effect has been established both experimentally ${ }^{7-12}$ and theoretically. ${ }^{6,13-20}$ When large spheres approach each other up to a distance smaller than the diameter of a smaller sphere, the latter is expelled from the gap. This also holds for mixtures of polymers and hard spheres, if the former may be considered as penetrable hard spheres. ${ }^{21,22}$ In the binary hard-sphere case the correlations between the smaller spheres induce, in addition to the attraction at short separations, an oscillatory depletion potential for larger distances between the big spheres. ${ }^{6}$

Colloidal mixtures of hard spheres with hard rods give also an entropically driven phase-separation according to experiments ${ }^{23-25}$ as well as theory. ${ }^{26-29}$ When placed between two spheres, the orientational entropy of the rods decreases. The consequent pressure deficit leads to an effective attraction of the spheres, which may lead to phase separation. For this system the depletion potential was measured directly $^{30}$ and was found in excellent agreement with theory. ${ }^{31-33}$ For mixtures of colloidal spheres and plates, depletion may be expected analogously and as indeed observed experimentally ${ }^{34}$ and theoretically. ${ }^{35,36}$
Many of the theoretical approaches to mixtures of spheres with depletion agents stem from the so-called freevolume theory, ${ }^{37}$ where the volume excluded to a single depletion agent plays a major role. The excluded volume had been derived from scaled-particle theory (SPT). ${ }^{38-40}$ Since this excluded volume is basically only a function of the integrated mean curvature, surface area, and volume of the depletion agent as well as the sphere, the theory may be expressed in terms of these so-called characteristic geometrical measures to arrive at a general version of the theory. This is very reminiscent of Rosenfeld's fundamental-measure theory (FMT) ${ }^{41-44}$ In fact, it has been shown ${ }^{45}$ that a density functional description of polymer/sphere mixtures using FMT yields the identical bulk free energy as free-volume theory. These connections make it sensible to generalize the free-volume theory in terms of characteristic measures by means of FMT as well.

Here we first briefly summarize the essence of freevolume theory. Subsequently, we derive general expressions for the free volume and the pressure of the depletion agents by means of SPT as well as FMT. We compare these quantities to numerical simulations. Finally we will demonstrate that the generalized theories make it easy to compare different shapes for depletion agents and derive phase diagrams for mixtures of different shapes yet unexplored. Hence, in this paper we provide a general "recipe" or "cook book" to free-volume theory.

\section{FREE-VOLUME THEORY}

Consider a system of $N_{s}$ hard spheres in a volume $V$ at temperature $T$ that is in equilibrium with a reservoir of deple- 
tion agents possessing a chemical potential $\mu_{d}^{R}$. The appropriate thermodynamic state variable to describe this system is the semigrand potential

$$
\begin{aligned}
\Omega\left(N_{s}, \mu_{d}, V, T\right) & \equiv \Omega\left(N_{s}, \mu_{d} \rightarrow \infty, V, T\right)-\int_{-\infty}^{\mu_{d}} N_{d} d \mu_{d}^{\prime} \\
& =A^{\circ}\left(N_{s}, V, T\right)-\int_{-\infty}^{\mu_{d}} N_{d} d \mu_{d}^{\prime},
\end{aligned}
$$

where $A^{\circ}$ is the Helmholtz free energy of the pure, i.e., unperturbed, hard-sphere system. The subscript $s$ will henceforth refer to the colloidal spheres, whereas the index $d$ to the depletion agent.

At low volume fractions of depletion agents in the reservoir, the chemical potential is given by $\mu_{d}^{R}=\mu_{d}^{\circ}$ $+k_{B} T \ln \phi_{d}^{R}$, where $\phi_{d}=N_{d} v_{d} / V$ is the volume fraction of depletion agents and $v_{d}$ the particle volume. The work required to insert a depletion agent into a sea of spheres follows from Widom's insertion theorem. ${ }^{46}$ For sufficient dilution this theorem provides us with $\mu_{d}=\mu_{d}^{\circ}$ $+k_{B} T \ln \phi_{d} V /\left\langle V_{\text {free }}\right\rangle_{0}$, where $\left\langle V_{\text {free }}\right\rangle_{0}$ is the (initially) available, i.e., free volume for the depletion agent in the sea of spheres. At equilibrium, $\mu_{d}^{R}=\mu_{d}$, we hence find for low volume fractions of depletion agents

$$
\phi_{d}^{R}=\frac{V}{\left\langle V_{\text {free }}\right\rangle_{0}} \phi_{d} \equiv \frac{\phi_{d}}{\alpha},
$$

which defines the free-volume fraction $\alpha$. Insertion of this equilibrium relation as well as the Gibbs-Duhem relation at constant temperature $T, d \mu_{d}=d \mu_{d}^{R}=d p^{R} / \phi_{d}^{R}$, into Eq. (1) amounts to

$$
\begin{aligned}
\Omega\left(N_{s}, \mu_{d}, V, T\right) & =A^{\circ}\left(N_{s}, V, T\right)-\int_{0}^{p^{R}} \frac{\phi_{d} V}{\phi_{d}^{R}} d p^{R} \\
& =A^{\circ}\left(N_{s}, V, T\right)-\int_{0}^{p^{R}}\left\langle V_{\text {free }}\right\rangle_{0} d p^{R} .
\end{aligned}
$$

If we furthermore assume the mean-field approximation that the volume initially available to the depletion agents is independent of the reservoir pressure, we find

$$
\begin{aligned}
\Omega\left(N_{s}, \mu_{d}, V, T\right) & =A^{\circ}\left(N_{s}, V, T\right)-p^{R}\left\langle V_{\text {free }}\right\rangle_{0} \\
& =A^{\circ}\left(N_{s}, V, T\right)-\alpha p^{R} V .
\end{aligned}
$$

Although Eq. (4) is strictly only valid in the limits $\phi_{d} \rightarrow 0$ and $\phi_{d}^{R} \rightarrow 0$, it turns out to be a good first-order approximation for any finite volume fraction of depletion agents.

We are interested in the phase behavior of mixtures of colloidal spheres and depletion agents. To this end we look for the equilibrium between two phases I and II, which is found when the thermodynamic conditions $\mu_{s}^{\mathrm{I}}=\mu_{s}^{\mathrm{II}}$ and $p^{\mathrm{I}}$ $=p^{\mathrm{II}}$ are met. The free-volume expressions for the chemical potential of the spheres, $\mu_{s}$, and the system's pressure $p$ follow from the total differential $d \Omega=-S d T-p d V+\mu_{s} d N_{s}$ $-N_{d} d \mu_{d}$ and Eq. (4) as

$$
\begin{aligned}
p=-\left(\frac{\partial \Omega}{\partial V}\right)_{T, N_{s}, \mu_{d}} & =p^{\circ}-p^{R}\left[\alpha+V\left(\frac{\partial \alpha}{\partial V}\right)\right] \\
& =p^{\circ}-p^{R}\left[\alpha-\phi_{s}\left(\frac{\partial \alpha}{\partial \phi_{s}}\right)\right]
\end{aligned}
$$

and

$$
\begin{aligned}
\mu_{s}=\left(\frac{\partial \Omega}{\partial N_{s}}\right)_{V, T, \mu_{d}} & =\mu_{s}^{\circ}-p^{R} V\left(\frac{\partial \alpha}{\partial \phi_{s}}\right)\left(\frac{\partial \phi_{s}}{\partial N_{s}}\right) \\
& =\mu_{s}^{\circ}-p^{R} v_{s}\left(\frac{\partial \alpha}{\partial \phi_{s}}\right) .
\end{aligned}
$$

The index ${ }^{\circ}{ }^{\circ}$ refers to the unperturbed hard-sphere system at a given volume fraction of spheres, $\phi_{s}$, and $v_{s}$ is the volume of a single sphere. The equation of state for a fluid of hard spheres is empirically well-described by the CarnahanStarling (CS) relation ${ }^{47}$

$$
\frac{p^{\circ} v_{s}}{k_{B} T}=\frac{\phi_{s}\left(1+\phi_{s}+\phi_{s}^{2}-\phi_{s}^{3}\right)}{\left(1-\phi_{s}\right)^{3}} \quad(\mathrm{CS}) .
$$

Omitting the cubic term in the numerator results in the Percus-Yevick (PY) compressibility equation of state. ${ }^{48}$ Using the Maxwell relation $\left(\partial \mu_{s} / \partial V\right)_{T}=-\left(\partial p / \partial N_{s}\right)_{T}$ or, alternatively,

$$
\left(\frac{\partial \mu_{s}}{\partial \phi_{s}}\right)_{T}=\frac{v_{s}}{\phi_{s}}\left(\frac{\partial p}{\partial \phi_{s}}\right)_{T},
$$

the chemical potential can be found from the CS pressure as

$$
\frac{\mu_{s}^{\circ}}{k_{B} T}=\ln \phi_{s}+\frac{\phi_{s}\left(8-9 \phi_{s}+3 \phi_{s}^{2}\right)}{\left(1-\phi_{s}\right)^{3}} \quad(\mathrm{CS}) \text {. }
$$

Analogously, for PY the chemical potential reads

$$
\frac{\mu_{s}^{\circ}}{k_{B} T}=\ln \frac{\phi_{s}}{1-\phi_{s}}+\frac{\phi_{s}\left(7-\frac{13}{2} \phi_{s}+\frac{5}{2} \phi_{s}^{2}\right)}{\left(1-\phi_{s}\right)^{3}} \quad(\mathrm{PY}) \text {. }
$$

For the hard-sphere crystal we employ the simple yet accurate equation of state given by ${ }^{49}$

$$
\frac{p^{\circ} v_{s}}{k_{B} T}=\frac{3 \phi_{s} \phi_{c p}}{\phi_{c p}-\phi_{s}}
$$

where $\phi_{c p}=\pi \sqrt{2} / 6 \approx 0.74$ is the volume fraction at closed packing of spheres. Thermodynamic integration results for the chemical potential in

$$
\frac{\mu_{s}^{\circ}}{k_{B} T}=2.1306+3 \ln \left(\frac{\phi_{s} \phi_{c p}}{\phi_{c p}-\phi_{s}}\right)+\frac{3 \phi_{c p}}{\phi_{c p}-\phi_{s}} .
$$

The integration constant is derived from the absolute free energy of a hard-sphere crystal from Monte Carlo simulations $^{50}$ for $\phi_{s}=0.576$.

With the expressions for the pure hard spheres at hand, we still require the free-volume fraction $\alpha$ and the reservoir pressure $p^{R}$ to establish phase equilibria. These will be derived in general geometric terms in the next two sections for both scaled-particle theory and fundamental-measure theory, respectively. 


\section{SCALED-PARTICLE THEORY}

\section{A. Free-volume fraction $\alpha$}

In previous papers on free-volume theory, the freevolume fraction $\alpha$ was determined from the chemical potential of the depletion agent by means of scaled-particle theory (SPT) ${ }^{38-40}$ Inserting a depletion agent in a sea of spheres adds, next to the mixing entropy, an insertion work term $W$ to that chemical potential,

$$
\mu_{d}=\mu_{d}^{\circ}+k_{B} T \ln \phi_{d}+W .
$$

Note that the work $W$ is equivalent to the excess chemical potential $\mu_{d}^{\mathrm{ex}}$ of the colloidal spheres in the dilute limit $\phi_{d}$ $\rightarrow 0$. On the other hand, it follows from the aforementioned insertion theorem ${ }^{46}$ that

$$
\mu_{d}=\mu_{d}^{\circ}+k_{B} T \ln \phi_{d}-k_{B} T \ln \alpha .
$$

The required work to insert a depletion agent in between spheres therefore amounts to $W=-k_{B} T \ln \alpha$ or, alternatively,

$$
\alpha=\exp \left(-W / k_{B} T\right)=\exp \left(-\mu_{d}^{\mathrm{ex}} / k_{B} T\right) .
$$

Looking for the free-volume fraction $\alpha$ is therefore equivalent to finding an expression for $W$. In order to determine the insertion work $W$, the scaled-particle theory considers two size limits. To that end, we scale each dimension $i$ of the depletion agent with a parameter $\lambda_{i}$.

For the limit $\lambda_{i} \ll 1$ the small (scaled) depletion agents may be considered to be point particles and the overlap volume between the spheres is therefore negligible. Hence, in that limit the volume available to the depletion agent is $V$ $-N_{s} \omega\left(\left\{\lambda_{i}\right\}, \sigma\right)$, where $\omega\left(\left\{\lambda_{i}\right\}, \sigma\right)$ is the volume around a sphere of diameter $\sigma$ that is excluded to the center of a depletion agent. Thus, the free-volume fraction is $\alpha=1$ $-\rho_{s} \omega\left(\left\{\lambda_{i}\right\}, \sigma\right)$, where $\rho_{s}$ is the number density of spheres. Using Eq. (15) it follows that

$$
W\left(\lambda_{i} \ll 1\right)=-k_{B} T \ln \left(1-\rho_{s} \omega\left(\left\{\lambda_{i}\right\}, \sigma\right)\right) .
$$

On the other hand, if $\lambda_{i} \gg 1$, the work required to insert a large (scaled) depletion agent between the spheres will, to leading order, be the volume work to create a cavity that is large enough to hold one depletion agent of volume $v_{d}$. Hence,

$$
W\left(\left\{\lambda_{i}\right\} \gg 1\right)=p v_{d} .
$$

The essence of the scaled-particle theory is that we may add both limits. To that end we expand $W$ in the limit $\lambda_{i}$ $\ll 1$ around $\lambda_{i}=0$ up to second order, representing a "surface area" and "radius" of the cavity of the inserted particle. ${ }^{40}$ The cubic term in $W$ is found from $\lambda_{i} \gg 1$. Hence,

$$
\begin{aligned}
W\left(\left\{\lambda_{i}\right\}\right)= & W(0)+\sum_{i}\left(\frac{\partial W}{\partial \lambda_{i}}\right)_{0} \lambda_{i} \\
& +\frac{1}{2} \sum_{i} \sum_{j}\left(\frac{\partial^{2} W}{\partial \lambda_{i} \partial \lambda_{j}}\right)_{0} \lambda_{i} \lambda_{j}+W\left(\left\{\lambda_{i}\right\} \gg 1\right) .
\end{aligned}
$$

Both summations over $i$ and $j$ run over all dimensions of the depletion agent. For spheres or infinitely thin objects $i=j$ $=1$, whereas for most finite sized objects, e.g., bodies of revolution, $\{i, j\}=1,2$. For some objects, like a bar or a general ellipsoid, $\{i, j\}=1,2,3$.

When we scale the depletion agent to the desired size by setting $\lambda_{i}=1$, we obtain from Eq. (18) using Eqs. (16) and (17)

$$
\begin{aligned}
\frac{W}{k_{B} T}= & -\ln \left(1-\rho_{s} v_{s}\right)+\left[\sum_{i}\left(\frac{\partial \omega}{\partial \lambda_{i}}\right)_{0}\right. \\
& \left.+\frac{1}{2} \sum_{i} \sum_{j}\left(\frac{\partial^{2} \omega}{\partial \lambda_{i} \lambda_{j}}\right)_{0}\right] \frac{\rho_{s}}{1-\rho_{s} v_{s}} \\
& +\frac{1}{2} \sum_{i} \sum_{j}\left(\frac{\partial \omega}{\partial \lambda_{i}}\right)_{0}\left(\frac{\partial \omega}{\partial \lambda_{j}}\right)_{0}\left(\frac{\rho_{s}}{1-\rho_{s} v_{s}}\right)^{2}+\frac{p v_{d}}{k_{B} T} .
\end{aligned}
$$

Here we used the fact that for infinitely small depletion agents $\left(\lambda_{i} \rightarrow 0\right)$ the excluded volume around the sphere is just the volume of the sphere itself, $\omega(0, \sigma)=v_{s}$. From this equation we can determine the free-volume fraction using Eq. (15), with any given expression for the system's pressure $p$. A thermodynamically consistent and convenient expression for the pressure is the PY compressibility equation in terms of characteristic measures [see Sec. III B, Eq. (36)],

$$
\frac{p}{k_{B} T}=\frac{\rho_{s}}{1-\rho_{s} v_{s}}+c_{s} a_{s}\left(\frac{\rho_{s}}{1-\rho_{s} v_{s}}\right)^{2}+\frac{a_{s}^{2} c_{s}^{2}}{3}\left(\frac{\rho_{s}}{1-\rho_{s} v_{s}}\right)^{3},
$$

where $v_{s}=\pi \sigma^{3} / 6$ is the volume, $a_{2}=\pi \sigma^{2}$ the surface area, and $c_{s}=\sigma / 2$ the integrated (over the surface) mean curvature of a sphere. Substitution of Eq. (20) into the insertion work, Eq. (19), gives for the free-volume fraction of Eq. (15),

$$
\begin{aligned}
\alpha= & (1-\phi) \exp \left[-A\left(\frac{\phi_{s}}{1-\phi_{s}}\right)-B\left(\frac{\phi_{s}}{1-\phi_{s}}\right)^{2}\right. \\
& \left.-C\left(\frac{\phi_{s}}{1-\phi_{s}}\right)^{3}\right],
\end{aligned}
$$

where $\phi_{s}=\rho_{s} v_{s}$ is the volume fraction of spheres and

$$
\begin{aligned}
& A v_{s}=\sum_{i}\left(\frac{\partial \omega}{\partial \lambda_{i}}\right)_{0}+\frac{1}{2} \sum_{i} \sum_{j}\left(\frac{\partial^{2} \omega}{\partial \lambda_{i} \partial \lambda_{j}}\right)_{0}+v_{d} \\
& B v_{s}^{2}=\frac{1}{2}\left[\sum_{i}\left(\frac{\partial \omega}{\partial \lambda_{i}}\right)_{0}\right]^{2}+c_{s} a_{s} v_{d} \\
& C v_{s}^{3}=\frac{a_{s}^{2} c_{s}^{2} v_{d}}{3}
\end{aligned}
$$

Let us consider the depletion agent to be a convex body with an Euler characteristic of $\chi_{d}=1$. Here we use for the Euler characteristic, the definition

$$
\chi_{d}=\frac{1}{4 \pi} \int_{\partial v_{d}} K(\vec{r}) d a_{s},
$$

and for the integrated mean curvature 
TABLE I. The characteristic geometrical measures for typical depletion agents: $c_{d}$ is the integrated (over the surface) mean curvature, $a_{d}$ the particle's surface area, and $v_{d}$ the depletion agent's volume.

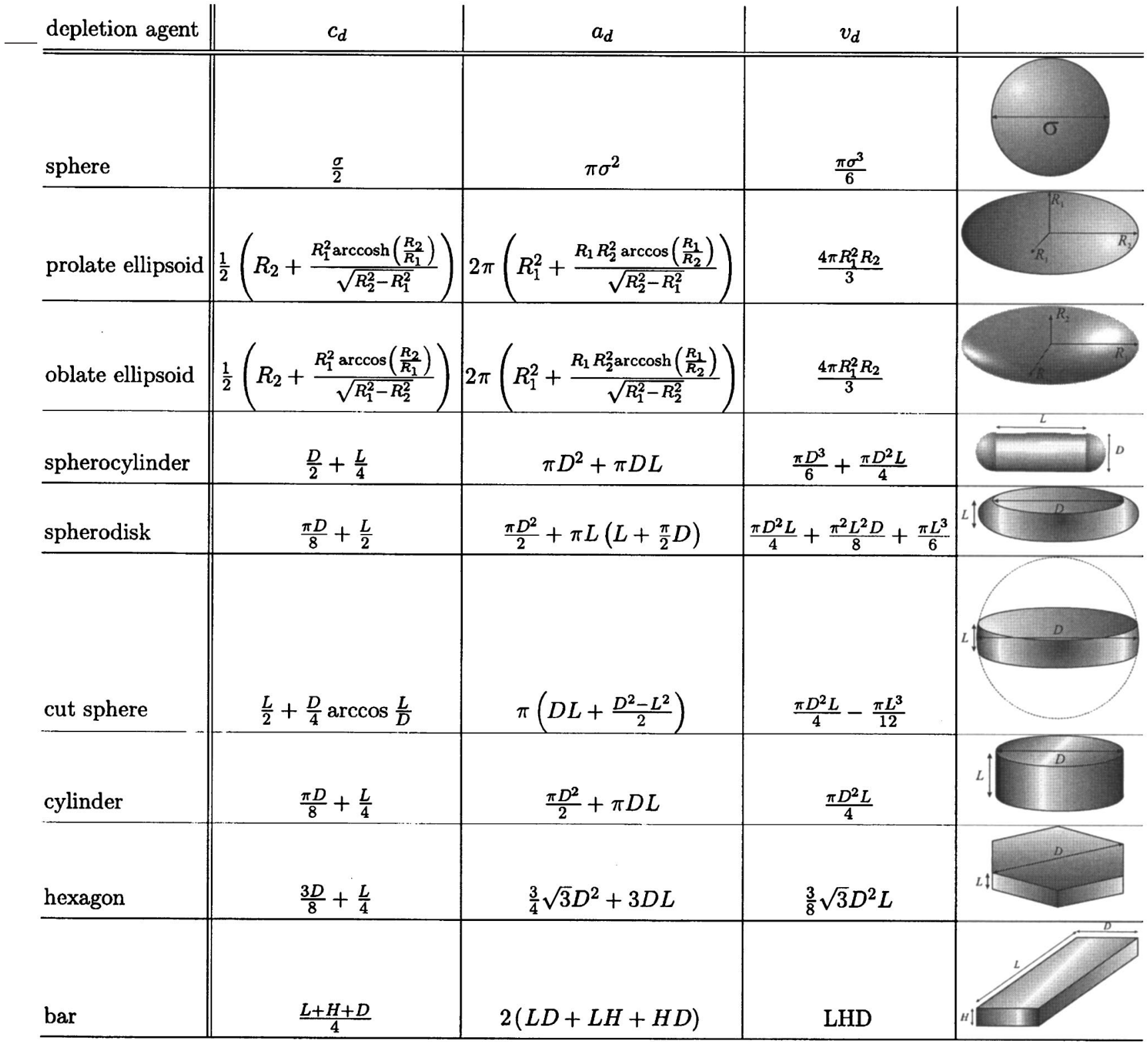

$$
c_{d}=\frac{1}{4 \pi} \int_{\partial v_{d}} H(\vec{r}) d a_{s},
$$

and $K(\vec{r})=\left[1 / R_{1}(\vec{r})\right]\left[1 / R_{2}(\vec{r})\right] \quad$ and $\quad H(\vec{r})=\frac{1}{2}\left(1 / R_{1}(\vec{r})\right.$ $\left.+1 / R_{2}(\vec{r})\right)$ are the local Gaussian and mean curvature, respectively, expressed in terms of the local principal radii of curvature $R_{1}(\vec{r})$ and $R_{2}(\vec{r})$. For bodies containing sharp edges, e.g., bars or hexagons, the curvature must be accounted for by replacing the edges by a parallel surface, e.g., a cylindrical-like "gutter" of a radius $\varepsilon$ in Eq. (26) and subsequently taking the limit $\varepsilon \rightarrow 0$. For sharp vertices the area of the parallel "cap" scales with $\varepsilon^{2}$, which makes their contribution vanish in the appropriate limit. Note that a procedure like this is necessary to recover the result that the Euler characteristic of such bodies equals unity. Expressions for the characteristic geometrical measures of some typical convex depletion agents are given in Table I.

The excluded volume of a convex body around a sphere is generally given by ${ }^{51}$

$$
\omega=v_{s}+a_{s} c_{d}+c_{s} a_{d}+v_{d},
$$

where $a_{d}$ is the surface area of a single depletion agent. Since only the characteristic measures of the depletion agent are scaled, the coefficients in the exponent of the free-volume fractions can be written as

$$
\begin{aligned}
& A v_{s}=a_{s} \sum_{i}\left(\frac{\partial c_{d}^{\mathrm{sp}}}{\partial \lambda_{i}}\right)_{0}+\frac{1}{2} c_{s} \sum_{i} \sum_{j}\left(\frac{\partial^{2} a_{d}^{\mathrm{sp}}}{\partial \lambda_{i} \partial \lambda_{j}}\right)_{0}+v_{d} \\
& B v_{s}^{2}=\frac{1}{2}\left[a_{s} \sum_{i}\left(\frac{\partial c_{d}^{\mathrm{sp}}}{\partial \lambda_{i}}\right)_{0}\right]^{2}+c_{s} a_{s} v_{d},
\end{aligned}
$$




$$
C v_{s}^{3}=\frac{a_{s}^{2} c_{s}^{2} v_{d}}{3}
$$

The index "sp" "refers to the fact that the characteristic dimensions of the depletion agent have been scaled. It is readily seen, e.g., from Table $\mathrm{I}$, that the scaled meancurvature $c_{d}^{\mathrm{sp}}$ is a first-order homogeneous function of the scaling parameters $\lambda_{i}$, whereas the scaled area $a_{d}^{\mathrm{sp}}$ is a homogeneous function of the second degree. Hence, the coefficients can finally be rewritten in terms of the characteristic geometrical measures of an unscaled particle

$$
\begin{aligned}
& A v_{s}=a_{s} c_{d}+c_{s} a_{d}+v_{d}, \\
& B v_{s}^{2}=\frac{1}{2}\left(a_{s} c_{d}\right)^{2}+c_{s} a_{s} v_{d}, \\
& C v_{s}^{3}=\frac{a_{s}^{2} c_{s}^{2} v_{d}}{3} .
\end{aligned}
$$

Note that for some bodies, e.g., spherocylinders ${ }^{52}$ the leading order term of the insertion work of a large particle, Eq. (17), is already of the order of $\lambda_{2}^{2}$, which has also been accounted for in the insertion work of small particles. This may in specific cases give incorrect results for $B$ in the expression for the free-volume fraction. However, the general expression for $B$ from SPT is given by Eq. (32).

It should be stressed that the PY compressibility expression for the pressure has been used explicitly to arrive at Eqs (31)-(33). Using any other pressure for the hard spheres and its chemical potential, $p^{\circ}$ and $\mu_{s}^{\circ}$, in Eqs (5) and (6) with Eq. (21) for the free-volume fraction, is inconsistent. This cannot be avoided since this is inherent to the scaled-particle treatment as it arises from Eq. (18).

\section{B. Reservoir pressure $p^{R}$}

Using the Maxwell relation, Eq. (8), the pressure of the reservoir can be determined from the chemical potential, which is given by Eq. (13). The insertion work $W$ is once more given by Eq. (19); however, now a scaled depletion agent is inserted into a sea of unscaled depletion agents. Hence, combining Eq. (13) with Eq. (19) provides us with

$$
\begin{aligned}
\frac{\mu_{d}}{k_{B} T}= & \frac{\mu_{d}^{\circ}}{k_{B} T}+\ln \left(\frac{\phi_{d}}{1-\phi_{d}}\right)+\frac{1}{v_{d}}\left[\sum_{i}\left(\frac{\partial \omega}{\partial \lambda_{i}}\right)_{0}\right. \\
& \left.+\frac{1}{2} \sum_{i} \sum_{j}\left(\frac{\partial^{2} \omega}{\partial \lambda_{i} \lambda_{j}}\right)_{0}\right]\left(\frac{\phi_{d}}{1-\phi_{d}}\right) \\
& +\frac{1}{2 v_{d}^{2}}\left[\sum_{i}\left(\frac{\partial \omega}{\partial \lambda_{i}}\right)_{0}\right]^{2}\left(\frac{\phi_{d}}{1-\phi_{d}}\right)^{2}+\frac{p v_{d}}{k_{B} T} .
\end{aligned}
$$

From the Maxwell relation, Eq. (8), we find after some elementary calculus

$$
\begin{aligned}
\frac{p v_{d}}{k_{B} T}= & \left(\frac{\phi_{d}}{1-\phi_{d}}\right)+\frac{1}{2 v_{d}}\left[\sum_{i}\left(\frac{\partial \omega}{\partial \lambda_{i}}\right)_{0}\right]\left(\frac{\phi_{d}}{1-\phi_{d}}\right)^{2} \\
& +\frac{1}{3 v_{d}^{2}}\left[\sum_{i}\left(\frac{\partial \omega}{\partial \lambda_{i}}\right)_{0}\right]^{2}\left(\frac{\phi_{d}}{1-\phi_{d}}\right)^{3} .
\end{aligned}
$$

If the inserted depletion agent is scaled, while the others are not, we derive using the same reasoning as in Sec. III A, with Eq. (27), that the reservoir equation of state is given by

$$
\begin{aligned}
\frac{p v_{d}}{k_{B} T}= & \left(\frac{\phi_{d}}{1-\phi_{d}}\right)+\frac{c_{d} a_{d}}{v_{d}}\left(\frac{\phi_{d}}{1-\phi_{d}}\right)^{2} \\
& +\frac{1}{3}\left(\frac{a_{d} c_{d}}{v_{d}}\right)^{2}\left(\frac{\phi_{d}}{1-\phi_{d}}\right)^{3} .
\end{aligned}
$$

This is indeed the expression as has previously been derived from scaled-particle theory. ${ }^{53}$

\section{FUNDAMENTAL-MEASURE THEORY}

Within fundamental-measure theory (FMT) the excess (over ideal gas) Helmholtz free-energy density $\Phi$ depends on so-called weighted densities $n_{i}$ and $i$ counts four scalarweighted densities and two vector-weighted densities. In the bulk limit in which the densities of all components are constant, the scalar-weighted densities simplify to the SPT variables while the vector-weighted densities vanish.

Following Schmidt et al. ${ }^{45}$ we distinguish in the following between the weighted densities of colloidal spheres and of depletion agents $n_{i}^{s}$ and $n_{i}^{d}$, respectively. By linearizing $\Phi$ in the density of depletion agents around vanishing densities of all depletion agents, we obtain

$$
\Phi^{\mathrm{AO}}\left(\left\{n_{i}^{s}, n_{i}^{d}\right\}\right)=\Phi\left(\left\{n_{i}^{s}\right\}\right)+\sum_{k=0}^{3} \frac{\partial \Phi\left(\left\{n_{i}^{s}\right\}\right)}{\partial n_{k}^{s}} n_{k}^{d} .
$$

Here we used the SPT variables of the $N$-component mixture $^{42-44}$

$$
\begin{aligned}
& n_{3} \equiv \sum_{j=1}^{N} v_{j} \rho_{j}, \quad n_{2} \equiv \sum_{j=1}^{N} a_{j} \rho_{j}, \\
& n_{1} \equiv \sum_{j=1}^{N} c_{j} \rho_{j}, \quad n_{0} \equiv \sum_{j=1}^{N} \chi_{j} \rho_{j},
\end{aligned}
$$

with the fundamental geometrical measures of the particles of component $j$, namely, the volume $v_{j}$, the surface area $a_{j}$, and the integrated (over the surface) mean curvature $c_{j}$, Eq. (26). The last term $n_{0}$ contains the Euler characteristics $\chi_{j}$, which are equal to unity for all particles that are topological equivalent to spheres, i.e., have no holes [cf. Eq. (25)]. This is the case for the depletion agents which we consider here. Equation (37) is our key equation and all FMT results we present here will use it as a starting point. It is the structure of this expression and the interpretation of its terms that allows one to generalize the free-volume theory to all kinds of situations with great ease including a different underlying thermodynamic theory and different shapes of the particles. It also becomes apparent from the structure of Eq. (37) that by choosing a particular Helmholtz excess free-energy density $\Phi$ for a mixture, the theoretical description of both the colloidal spheres and the depletion agents are prescribed. Note that all components are treated in the canonical ensemble, i.e., the system densities $\rho_{j}$ of all components are fixed. Rewriting Eq. (37) into the variables $\phi_{s}$ and $\rho_{d}$, 
TABLE II. The thermodynamic coefficients $p, \gamma, \kappa$, and $\bar{\kappa}$, which enter the free-volume fraction $\alpha$, Eq. (43), for both the PY and CS approach. $\phi_{s}, \rho_{s}$, and $\sigma$ are the packing fraction, the density, and the diameter of the colloidal spheres.

\begin{tabular}{ccc}
\hline \hline & $\mathrm{PY}$ & $\mathrm{CS}$ \\
\hline$p / k_{B} T$ & $\rho_{s}\left(1+\phi_{s}+\phi_{s}^{2}\right) /\left(1-\phi_{s}\right)^{3}$ & $\rho_{s}\left(1+\phi_{s}+\phi_{s}^{2}-\phi_{s}^{3}\right) /\left(1-\phi_{s}\right)^{3}$ \\
$\gamma / k_{B} T$ & {$\left[3 \phi_{s}\left(2+\phi_{s}\right)\right] /\left[2 \pi \sigma^{2}\left(1-\phi_{s}\right)^{2}\right]$} & {$\left[3 \phi_{s}\left(2-\phi_{s}\right)\right] /\left[\left(1-\phi_{s}\right)^{2} \pi \sigma^{2}\right]$} \\
& & $+3 \ln \left(1-\phi_{s}\right) / \pi \sigma^{2}$ \\
$\kappa / k_{B} T$ & $6 \phi_{s} / \sigma\left(1-\phi_{s}\right)$ & $6 \phi_{s} / \sigma\left(1-\phi_{s}\right)$ \\
$\bar{\kappa} / k_{B} T$ & $-\ln \left(1-\phi_{s}\right)$ & $-\ln \left(1-\phi_{s}\right)$ \\
\hline \hline
\end{tabular}

$$
\Phi^{\mathrm{AO}}\left(\phi_{s}, \rho_{d}\right)=\Phi\left(\phi_{s}\right)-\rho_{d} \ln \left(\alpha\left(\phi_{s}\right)\right)
$$

with the FMT expression for the free-volume fraction,

$$
\begin{aligned}
-\ln \left(\alpha\left(\phi_{s}\right)\right) & \equiv \sum_{k=0}^{3} \frac{\partial \Phi\left(\left\{n_{i}^{s}\right\}\right)}{\partial n_{k}^{s}} \frac{n_{k}^{d}}{\rho_{d}} \\
& =\frac{p v_{d}}{k_{B} T}+\frac{\gamma a_{d}}{k_{B} T}+\frac{\kappa c_{d}}{k_{B} T}+\frac{\bar{\kappa} \chi_{d}}{k_{B} T},
\end{aligned}
$$

where we used the generalized geometrical interpretation of the depletion agent weights. The thermodynamic coefficients of the colloidal system corresponding to the geometrical measures of the depletion agent are $p$ the pressure of the colloidal spheres, $\gamma$ the surface tension of the colloids at a planar hard wall, and $\kappa$ and $\bar{\kappa}$ the bending rigidities, which account for curvature effects-see Table II. Note that there is a simple thermodynamic interpretation of Eq. (40): $\alpha$ $=\lim _{p_{d} \rightarrow 0} \exp \left(-\mu_{d}^{\mathrm{ex}} / k_{B} T\right)$, where $\mu_{d}^{\mathrm{ex}}=W$ is the excess chemical potential of a single depletion agent in a sea of colloids, or in terms of SPT the reversible work of inserting a single particle of the depletion agent into a sea of spheres. If the interaction between the depletion agent and the colloids is considered to be hard, as we do here, then the excess chemical potential is the reversible work needed to create a cavity large enough to hold the depletion agent. This work depends, of course, on the shape of the cavity. There is a contribution that describes the work against the pressure of the colloids to create a cavity of volume $v_{d}$. This procedure creates also an interface between the depletion agent and the colloids with a surface area $a_{d}$, which is accounted for by the surface tension. However, the surface of the depletion agent is in general curved which has also been taken into account by the bending rigidities.

The simple form of Eq. (41) is appealing because it separates the thermodynamics of the colloidal system from the geometrical properties of the depletion agents. It has been shown numerically, using density functional theory and Monte Carlo simulations, that Eq. (41) accounts very accurately for the work to insert a single complex-shaped object into a bulk system. ${ }^{54-56}$

By transforming Eq. (39) into the semigrand canonical ensemble, in which we treat the colloids in the canonical and the depletion agents in the grand canonical ensemble, we obtain [cf. Eq. (4)]

$$
\Phi\left(\phi_{s}, \rho_{d}^{R}\right)=\Phi\left(\phi_{s}\right)-\rho_{d}^{R} \alpha\left(\phi_{s}\right),
$$

where we made use of the fact that the reservoir density of the depletion agent, $\rho_{d}^{R}$, is related to the canonical (or system) density by the factor $\alpha$. The general expression for $\alpha$ from FMT reads

$$
\alpha\left(\phi_{s}\right)=\left(1-\phi_{s}\right) \exp \left(-\left\{p v_{d}+\gamma a_{d}+\kappa c_{d}\right\} / k_{B} T\right),
$$

where we have used the fact that the Euler characteristic $\chi_{d} \equiv 1$ and $\bar{\kappa} / k_{B} T=-\ln \left(1-\phi_{s}\right)$. It is interesting to notice that the term $\bar{\kappa} \chi_{d}$ allows for an alternative interpretation of Eq. (16). Within SPT it accounts for the work of inserting a pointlike particle into a sea of spheres. From the morphometric point of view ${ }^{54-56}$ this contribution to the insertion work is independent of the size of the particle. It is the only term of $\mu_{d}^{\mathrm{ex}}$ or, alternatively, $W$ in the limit of pointlike particles, because all other characteristic geometrical measures of a point particle vanish, while the Euler characteristic remains unity.

In order to fully specify the FMT expression for $\alpha$, we have to prescribe the thermodynamic coefficients $p, \gamma$, and $\kappa$. These coefficients are related to the free-energy density $\Phi\left(\left\{n_{i}\right\}\right)$ of the colloidal spheres via Eq. (40). We get

$$
\frac{p}{k_{B} T}=\frac{\partial \Phi}{\partial n_{3}}, \quad \frac{\gamma}{k_{B} T}=\frac{\partial \Phi}{\partial n_{2}}, \quad \frac{\kappa}{k_{B} T}=\frac{\partial \Phi}{\partial n_{1}}, \quad \frac{\bar{\kappa}}{k_{B} T}=\frac{\partial \Phi}{\partial n_{0}} .
$$

One possible choice for $\Phi\left(\left\{n_{i}\right\}\right)$ is the expression for the excess free-energy density which results from Rosenfeld's FMT functional ${ }^{42}$ in the bulk limit (cf. Table II),

$$
\frac{\Phi\left(\left\{n_{i}\right\}\right)}{k_{B} T}=-n_{0} \ln \left(1-n_{3}\right)+\frac{n_{1} n_{2}}{1-n_{3}}+\frac{n_{2}^{3}}{24 \pi\left(1-n_{3}\right)^{2}} \text {. }
$$

The pressure underlying Rosenfeld's FMT functional is the Percus-Yevick compressibility equation of state, which makes this application of FMT comparable to the SPT approach. The free-volume fraction can also be rewritten in the form of Eq. (21), with the parameters $A, B$, and $C$ given now by

$$
\begin{aligned}
& A v_{s}=a_{s} c_{d}+c_{s} a_{d}+v_{d}, \\
& B v_{s}^{2}=\frac{a_{s}^{2} a_{d}}{8 \pi}+c_{s} a_{s} v_{d}, \\
& C v_{s}^{3}=\frac{a_{s}^{2} c_{s}^{2} v_{d}}{3} .
\end{aligned}
$$

For the corresponding reservoir pressure of the depletion agents we find from Eqs. (38) and (45),

$$
\begin{aligned}
\frac{p v_{d}}{k_{B} T}= & \left(\frac{\phi_{d}}{1-\phi_{d}}\right)+\frac{c_{d} a_{d}}{v_{d}}\left(\frac{\phi_{d}}{1-\phi_{d}}\right)^{2} \\
& +\left(\frac{a_{d}^{3}}{12 \pi v_{d}^{2}}\right)\left(\frac{\phi_{d}}{1-\phi_{d}}\right)^{3} .
\end{aligned}
$$

It is straightforward to modify the FMT approach in order to incorporate different thermodynamics, i.e., a freevolume theory that is based entirely on a more accurate 
modified Carnahan-Starling equation ${ }^{57}$ (MCSL) rather than the PY equation of state. In the bulk the excess free-energy density based on the MCSL (Ref. 57) equation of state [cf. Eq. (7)],

$$
\frac{p_{\mathrm{MCSL}}}{k_{B} T}=\frac{n_{0}}{1-n_{3}}+\frac{n_{1} n_{2}}{\left(1-n_{3}\right)^{2}}+\frac{n_{2}^{3}\left(1-\frac{1}{3} n_{3}\right)}{12 \pi\left(1-n_{3}\right)^{3}},
$$

is given by ${ }^{58,59}$

$$
\begin{aligned}
\frac{\Phi\left(\left\{n_{i}\right\}\right)}{k_{B} T}= & -n_{0} \ln \left(1-n_{3}\right)+\frac{n_{1} n_{2}}{1-n_{3}} \\
& +\frac{n_{2}^{3}\left[n_{3}+\left(1-n_{3}\right)^{2} \ln \left(1-n_{3}\right)\right]}{36 \pi n_{3}^{2}\left(1-n_{3}\right)^{2}} .
\end{aligned}
$$

The pressure $p$ is that due to Carnahan-Starling, Eq. (50), or for a one-component system, Eq. (7). Note that this is thermodynamically slightly inconsistent with $p / k_{B} T$ $=\partial \Phi / \partial n_{3}$, but numerically both results are very close ${ }^{58}$ The results for a one-component fluid of colloidal spheres of diameter $\sigma$, density $\rho_{s}$ and packing fraction $\phi_{s}$ are summarized in Table II both for the FMT approach based on the thermodynamics of PY and CS.

Especially if the coexistence between a fluid and a solid phase is considered, it is important to use the expression for the free volume which corresponds to the CS equation of state. In the case of the pure hard-sphere system, the coexisting densities of the hard-sphere fluid and solid predicted by the CS equation of state are significantly improved over that derived from a PY description of the hard-sphere system. $^{58}$

Note that FMT is from the outset a theory for mixtures of particles. Therefore it is possible to describe mixtures of spheres and nonspherical depletion agents on equal footing by using the full free-energy density of the mixture, Eq. (45) or Eq. (51), and hence avoiding the linearized form, Eq. (37). In the present context we wish to improve the understanding of the free-volume approach, which is based on Eq. (37), as pointed out before.

\section{RESULTS AND DISCUSSION}

\section{A. Comparison SPT vs FMT}

The similarity between the SPT and FMT methods to free-volume theory is striking. However, there are small differences that may influence the accuracy of the calculations. Here we first consider the differences in the free-volume fraction and the reservoir pressure of a mixture of large spheres with small depletion agents in PY approximation.

Within the PY approach the free-volume fraction of both SPT and FMT take the form of Eq. (21). A [Eqs. (31) and (46)] and $C$ [Eqs. (33) and (48)] are exactly the same. However, the first terms on the right-hand side of $B$ in Eqs. (32) and (47) differ. The FMT result can be recovered from SPT by omitting the second derivative of the excluded volume for $(i=j) \wedge(i>1)$. This has for instance been done previously for spherocylinders ${ }^{52}$ since the leading order term of the insertion work of a large particle, Eq. (17), is already of the order of $\lambda_{2}^{2}$. However, there is no obvious reason to do this in general. Note that for small spheres as depletion agents,
TABLE III. Comparison of the reduced third virial coefficient, $B_{3} / B_{2}^{2}$, for isotropic hard spherocylinders calculated from SPT and FMT with those from accurate numerical results (Ref. 62) for several aspect ratios.

\begin{tabular}{lccc}
\hline \hline$L / D$ & SPT & FMT & numerical \\
\hline 0 & $5 / 8$ & $5 / 8$ & 0.625 \\
5 & 0.4824 & 0.3239 & 0.4194 \\
10 & 0.4293 & 0.2203 & 0.3133 \\
$10^{2}$ & 0.3461 & 0.03176 & 0.0698 \\
$10^{3}$ & 0.3346 & 0.00331 & 0.0106 \\
$\infty$ & $1 / 3$ & 0 & $\cdots$ \\
\hline \hline
\end{tabular}

$\frac{1}{2} a_{s}^{2} c_{p}^{2}=\frac{1}{2} a_{s}^{2} \sigma^{2} / 4=(1 / 8 \pi) a_{s}^{2} a_{d}$, the scaled-particle approach yields the same result as fundamental-measure theory.

Also the reservoir pressure of the depletion agents, Eqs. (36) and (49), has the same structure for both approaches. However, the prefactor of the cubic terms differ, as already has been pointed out by Rosenfeld. ${ }^{60}$ It is once more easily seen that for spheres, $a_{d} / 12 \pi=\sigma^{2} / 12=c_{d}^{2} / 3$, both methods yield the same PY compressibility equation of state.

Expansion of Eqs. (36) and (49) in the number density provides us with a virial series from which it is easily seen that the second virial coefficient reads for both SPT and FMT,

$$
B_{2}=v_{d}+c_{d} a_{d} .
$$

From Eq. (27) it is found that $B_{2}=\frac{1}{2} \omega$, which is the exact second virial coefficient. However, the third virial coefficient from SPT reads

$$
B_{3}=v_{d}^{2}+2 c_{d} a_{d} v_{d}+\frac{1}{3} c_{d}^{2} a_{d}^{2},
$$

whereas for FMT using PY,

$$
B_{3}=v_{d}^{2}+2 c_{d} a_{d} v_{d}+\frac{a_{d}^{3}}{12 \pi} .
$$

For spheres both reduce to the same, exact third virial coefficient: $B_{3}=10 v_{d}^{2}$.

Barker and Henderson ${ }^{53}$ state that Eq. (36) gives an accurate third virial coefficient for prolate spherocylinders and conjecture from this fact that SPT gives the correct $B_{3}$ for all convex molecules. However, for spherocylinders the formal SPT approach gives an incorrect result for $L / D \rightarrow \infty$, as can be found in Table III. Onsager has shown from geometric arguments ${ }^{61}$ that for $L \gg D$ the reduced third virial coefficient of the isotropic phase should scale as

$$
\frac{B_{3}}{B_{2}^{2}} \propto \frac{D}{L} \ln \frac{L}{D} .
$$

Hence, this ratio should vanish for $L / D \rightarrow \infty$. This result is recovered by FMT and yields a fair approximation for the exact result ${ }^{62}$ for different size ratios, as can be seen from Table III. The incorrect behavior of the generalized scaledparticle theory for spherocylinders must be due to the fact that we have included a second-order $\lambda_{2}$ term of the diameter $D$ in the series expansion of the limit of infinitely small spherocylinders. However, the term of the order $\lambda_{2}^{2}$ is also present in the insertion work of a large particle and has thus been omitted previously. ${ }^{52}$ 
TABLE IV. Comparison of the reduced second, $B_{2} / D^{3}$, and third virial coefficient, $B_{3} / B_{2}^{2}$, for isotropic hard cut spheres calculated from SPT and FMT with those from accurate numerical results (Refs. 63-66) for several aspect ratios.

\begin{tabular}{lcccccc}
\hline \hline & \multicolumn{2}{c}{$B_{2} / D^{3}$} & & \multicolumn{3}{c}{$B_{3} / B_{2}^{2}$} \\
\cline { 2 - 3 } \cline { 5 - 6 }$L / D$ & SPT/FMT & Numerical & & SPT & FMT & Numerical \\
\hline 0 & $\pi^{2} / 16$ & $\pi^{2} / 16$ & & $1 / 3=0.3333$ & $8 / 3 \pi^{2}=0.2701$ & 0.4447 \\
$1 / 20$ & 0.737771 & 0.736951 & & 0.339388 & 0.293838 & 0.6235481 \\
$1 / 15$ & 0.778170 & 0.794320 & & 0.343204 & 0.301909 & 0.7071306 \\
$1 / 10$ & 0.858983 & 0.855538 & & 0.352253 & 0.317510 & 0.8835688 \\
1 & $2 \pi / 3$ & $2 \pi / 3$ & $5 / 8$ & $5 / 8$ & $5 / 8$ \\
\hline \hline
\end{tabular}

Another comparison of the reservoir pressure with accurate numerical results can be made for cut spheres (see Table I). Using Eqs. (52) and (53) the reduced third virial coefficient of isotropic cut spheres may again be found from straightforward algebra. The numerical results of infinitely thin plates ${ }^{63,64}(L / D=0)$ can directly be compared to these analytical results. It is seen from Table IV that both theories give a slightly different result for the reduced third virial coefficient than the exact solution. The SPT expression is slightly less off than the one from FMT.

Numerical results for finite thicknesses are available for three aspect ratios. ${ }^{65,66}$ The second virial coefficients are slightly off from the numerical results, as shown in Table IV. This is due to the fact that the numerical results are actually polynomial fits to the simulations and not real virial coefficients. It can also be seen from Table IV that both SPT and FMT do not predict the third virial coefficients very well, although SPT is once more slightly less off. This makes the SPT and FMT expressions for plates poor approximations to determine the isotropic-nematic phase transitions (typically at $\left.N D^{3} / V \approx 4\right)$. However, in most applications of depletion agents mixed with large spheres, we are far away from this transition (typically $N D^{3} / V<2$ ) and both the FMT and SPT equation of state suffices. ${ }^{35}$ Note that the simulation data for the reduced third virial coefficient apparently goes through a maximum as a function of aspect ratio to go to the analytical result for spheres $(L / D=1)$.

\section{B. Mixtures of spheres and ellipsoids}

From the characteristic measures of ellipsoids of revolution in Table I, we can smoothly vary the depletion agent from infinitely thin rods via spheres to infinitely thin platelets in a gedanken experiment. A similar approach has been carried out previously for the depletion potential. ${ }^{67}$

Let us start with a mixture of spheres and a spherical depletion agent, i.e., $R_{1}=R_{2}$. If we now start to deform the shape of the depletion agent a little bit by decreasing $R_{1}$ while keeping the value of $R_{2}$ fixed, i.e., $R_{1}<R_{2}$, we get a prolate ellipsoid. The physics of the problem should not change at all and there is clear numerical evidence ${ }^{54-56}$ that the form of Eq. (41) remains valid. If we further decrease $R_{1}$, the shape of the depletion agent continuously approaches that of an infinitely thin rod of length $L=2 R_{2}$, for $R_{1} \rightarrow 0$. The geometrical measures of the ellipsoid indeed recover those of the infinitely thin rod, either represented by infinitely thin (sphero)cylinders $(D \rightarrow 0)$ or bars $(D=H \rightarrow 0)$.
Rods of finite thicknesses can be represented either by spherocylinders ${ }^{27}$ or prolate ellipsoids. The geometrical measures of these long prolate ellipsoids are quite different from those of spherocylindrical rods-the volume and the surface area, which vanish in the limit of zero thickness, and the integrate mean curvature increase faster for a spherocylinder than for an ellipsoid with the same thickness. As a result of the different shapes, the critical point of the fluid-fluid phase separation in the mixture of spheres and spherocylinders moves along a different path than that for depletion of spheres due to ellipsoids as the thickness of the rods is increased, as shown in Fig. 1 from FMT calculations. In a mixture of spheres and spherocylinders the critical point moves to significantly lower densities of the rods in the reservoir (solid line), which reflects the increasing attraction of the corresponding depletion potential. ${ }^{67}$ However, the critical point in the mixture of spheres and prolate ellipsoids (dashed line) first moves to higher packing fractions of the spheres, while the reservoir density of the rods remain almost un-

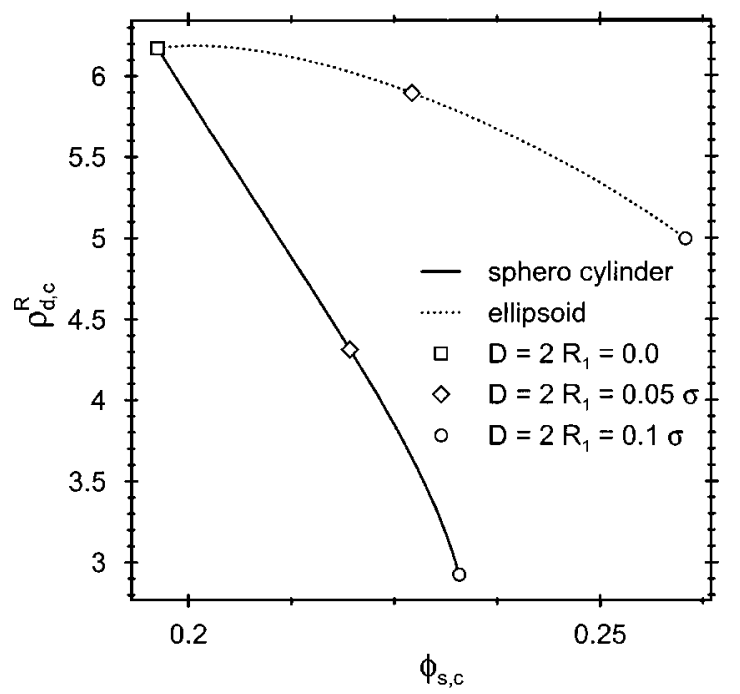

FIG. 1. The path of the critical point of the fluid-fluid phase separation in a mixture of spheres and rods as the thickness of the rods increases from $D$ $=R_{1}=0$ to $D=2 R_{1}=0.1 \sigma$ using FMT. The solid line denotes the path of the critical point for spherocylindrical rods, whereas the dashed line that for ellipsoidal rods. The symbols indicate that at small values of $\sigma$ the critical point for mixtures of spheres and ellipsoids moves mainly to higher values of $\phi_{s}$, while the densities (in the reservoir) of ellipsoids at the critical point remains roughly the same. In contrast to this, the critical point in a mixture of spheres and spherocylinders moves to lower densities of rods rapidly. 

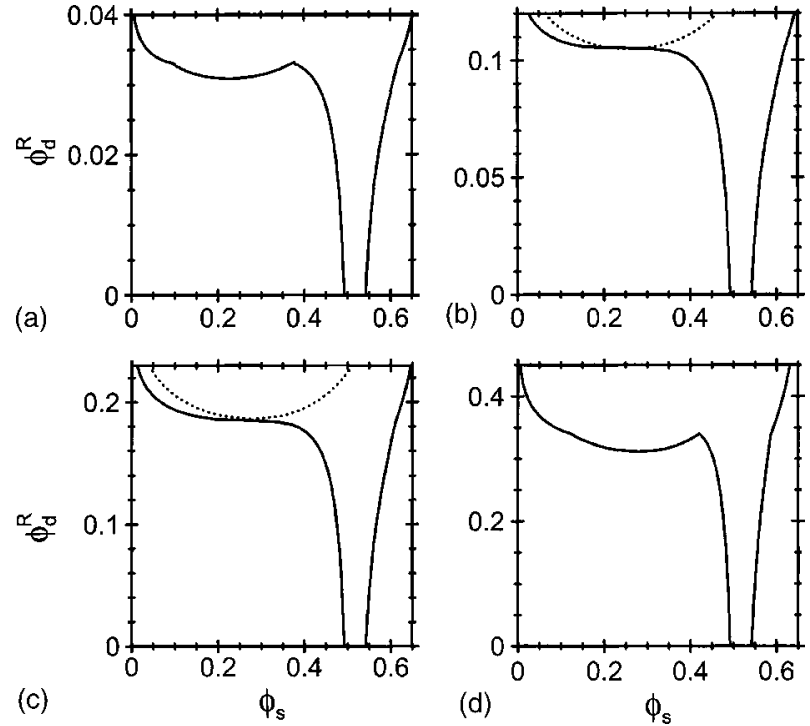

FIG. 2. The fluid-fluid phase separation in a mixture of spheres and ellipsoids can become metastable with respect to crystallization as the thickness of the rods is increased. For $R_{1}=0.025 \sigma$ the fluid-fluid phase separation is stable. As the thickness $R_{1}$ is increased, the phase separation becomes metastable (b) and (c), which corresponds to $R_{1}=0.05 \sigma$ and $R_{1}=0.075 \sigma$, respectively. If $R_{1}$ is further increased the fluid-fluid phase separation has to become stable again in (d), where $R_{1}=0.125 \sigma$.

changed. Moreover, only if the ellipsoids are thick enough the fluid-fluid phase separation will occur at lower densities of the rods.

Note that as the thickness of the ellipsoids increases, the fluid-solid binodal broadens and also moves to lower rod densities. Although increasing thickness leaves the topology of the phase diagram of the mixture of spheres and spherocylindrical rods unchanged, ${ }^{27}$ the fluid-fluid phase separation in the sphere/ellipsoid mixture becomes eventually metastable with respect to the crystallization. If we continue to increase $R_{1}$ further, the fluid-fluid phase separation must become stable again when the spherical shape of the depletion agent is recovered for $R_{1} \rightarrow R_{2}$. In Fig. 2 we show a sequence of phase diagrams for (a) $R_{1}=0.025 \sigma$, (b) $R_{1}=0.05 \sigma$, (c) $R_{1}=0.075 \sigma$, and (d) $R_{1}=0.125 \sigma$. This shows that the fluidfluid phase separation is indeed stable for $R_{1}<0.05 \sigma$, metastable for $0.05 \sigma<R_{1}<0.075 \sigma$, and becomes stable again for $R_{1}>0.075 \sigma$. In Fig. 2 the metastable fluid-fluid phase separation is denoted by dotted lines.

We may also deform a spherical depletion agent by decreasing $R_{2}$ while keeping $R_{1}$ constant, i.e., create an oblate ellipsoid. This way we can continuously change the shape from spherical to that of infinitely thin disks as we approach the limit $R_{2} \rightarrow 0$ and $R_{1} \rightarrow \frac{1}{2} D$. The characteristic measures of the ellipsoids indeed approach those of the infinitely thin disk, as can be deduced from spherodisks, cut spheres, and cylinders $(L \rightarrow 0)$ from Table I. Mixtures of spheres and plates will be scrutinized in the following section.

\section{Mixtures of spheres and plates}

An experimental study of depletion interaction in mixtures of colloidal silica spheres with silica-coated gibbsite platelets has recently been conducted. ${ }^{34}$ The phase behavior of such systems has been predicted by means of free-volume theory, ${ }^{35}$ where the platelets are mimicked as cylinders. Given the hexagonal nature of the gibbsite platelets, ${ }^{68}$ it seems legitimate to question whether cylinders give a fair representation or not. With our current general approach, we can easily check this accuracy. To that end, we apply the FMT approach to spherodisks, cylinders, cut spheres, and hexagons (cf. Table I) starting from the CS functional, Eq. (51).

By applying a certain reservoir density of the depletion agents, $\phi_{d}^{R}$, one can determine the coexisting sphere densities from $\mu_{s}^{\mathrm{I}}=\mu_{s}^{\mathrm{II}}$ and $p^{\mathrm{I}}=p^{\mathrm{II}}$, using Eqs. (5) and (6). Applying CS, Eqs. (7) and (9), for the pure sphere fluid we find a completely metastable fluid-fluid region, which is not displayed for the sake of clarity. Using Eqs. (11) and (12) in addition to CS for the pure sphere system, we find stable fluid-solid coexistence over the whole volume-fraction
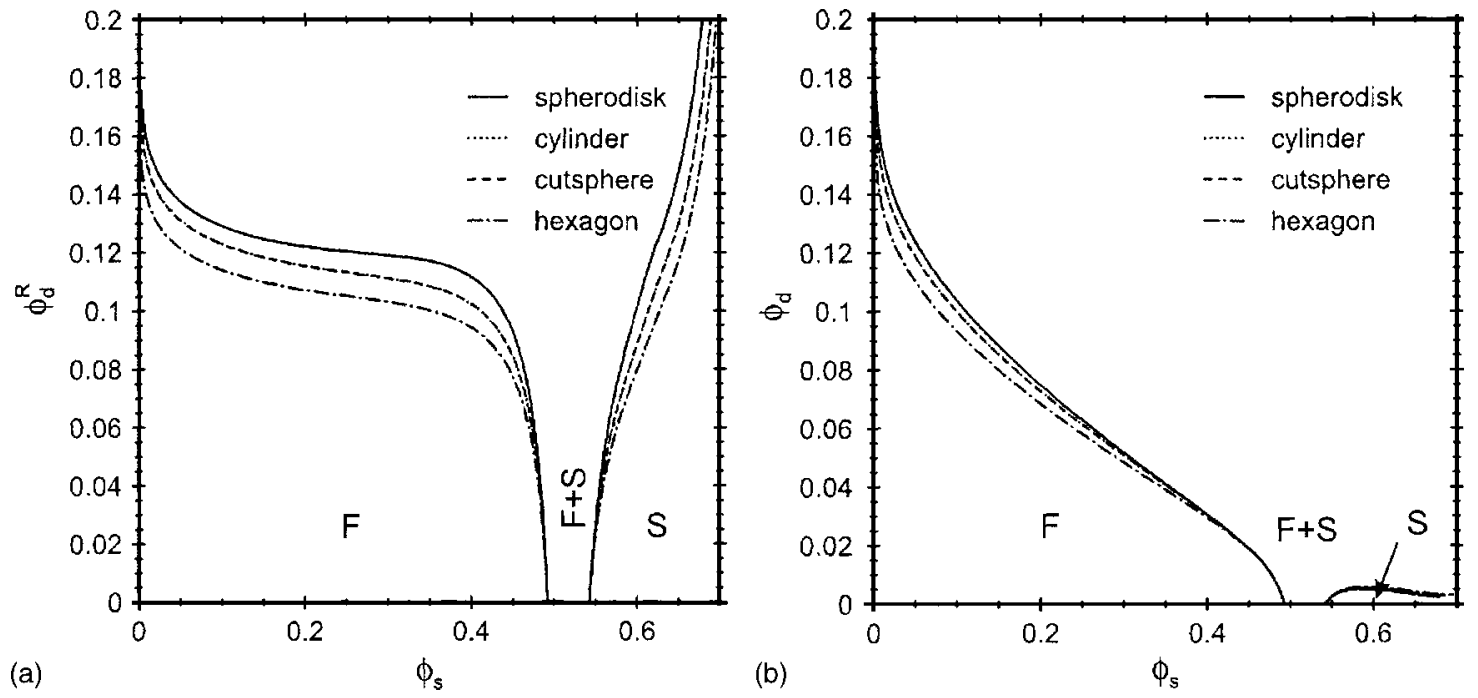

FIG. 3. The phase diagrams from the FMT/CS approach of a mixture of spheres and platelike mesogens, mimicked by spherodisks, cylinders, cut spheres, and hexagons (top to bottom, respectively) in both (a) the reservoir and (b) system representation. The measures are chosen to be such as in the experiments (Refs. $34,35)$, i.e., $D=200 \mathrm{~nm}, L=30 \mathrm{~nm}$, and $\sigma=700 \mathrm{~nm}$. 
range, as shown in Fig. 3(a). This phase diagram can be converted to system volume fractions of the plates using Eq. (2) and is shown in Fig. 3(b). Since this conversion via the free-volume fraction is not the same for fluid and solid phase, the tie lines are slanted rather than horizontal. For the sake of clarity the tie lines have also been omitted in Fig. 3 .

Because of its smaller values of the characteristic geometrical measures, the hexagon turns out to be the most effective depletion agent out of the four shapes. That is, phase separation is expected at relatively low volume fraction of hexagons. The values are, however, close to those predicted for other geometries. The difference between cylinders and cut spheres are indistinguishable for our choice of parameters. It is readily seen that for the aspect ratios applied here, the difference between their characteristic measures is negligible. The spherodisk has the largest values for the characteristic measures and is therefore the least effective of the selected geometries. When converted to experimentally accessible variables, Fig. 3(b), we reckon that the differences between the four geometries are likely to be too small to become important within the experimental error. This also comes across given the approximate nature of free-volume theory.

As compared to the phase diagram previously calculated from SPT, ${ }^{35}$ which give qualitatively the same results, the fluid-solid binodals are shifted slightly upwards using FMT/ CS. This turns out to be a generic difference between the two approaches, as will be elaborated in the following section.

\section{Mixtures of spheres and bars}

Goethite is a very common and stable iron oxide. Because of its brownish color goethite was already used by the caveman for their wall paintings and it is still used in contemporary paint industry. As mentioned in the Introduction, this alone makes a depletion study of mixtures of spherical particles and goethite worthwhile. Renewed interest in goethite, however, concerns the unusual magnetic properties of goethite. ${ }^{69,70}$ In aqueous environment these particles are charge stabilized and the interactions are not hard. However, the intrinsic excluded volume behavior may shortly be studied by coating the goethite particles with silica ${ }^{71}$ (cf. Fig. 4) and dispersing them in dimethylformamide. It now has become straightforward to study this intrinsic behavior for mixtures of spheres and bars using the recipe of our generalized free-volume theory.

From the characteristic measures of the bars (cf. Table I), the phase diagram can be calculated using FMT/CS like in the preceding section. Using the sizes of typical goethite nanorods, $L=230 \mathrm{~nm}, D=40 \mathrm{~nm}, H=8 \mathrm{~nm}$, and $\sigma=1000 \mathrm{~nm}$, FMT once more predict a metastable fluid-fluid binodal, indicated by the dotted line in Fig. 5. The stable fluid-solid binodal is drawn by the solid line.

The phase diagram can also be obtained from SPT, which effectively amounts to PY compressibility equation of state. For the same measures the fluid-fluid binodal is also predicted to be metastable, as indicated by the dashed line in Fig. 5. However, this gives unphysical results, since the solid phase may become denser than closed packed $\left(\phi_{s}>0.74\right)$

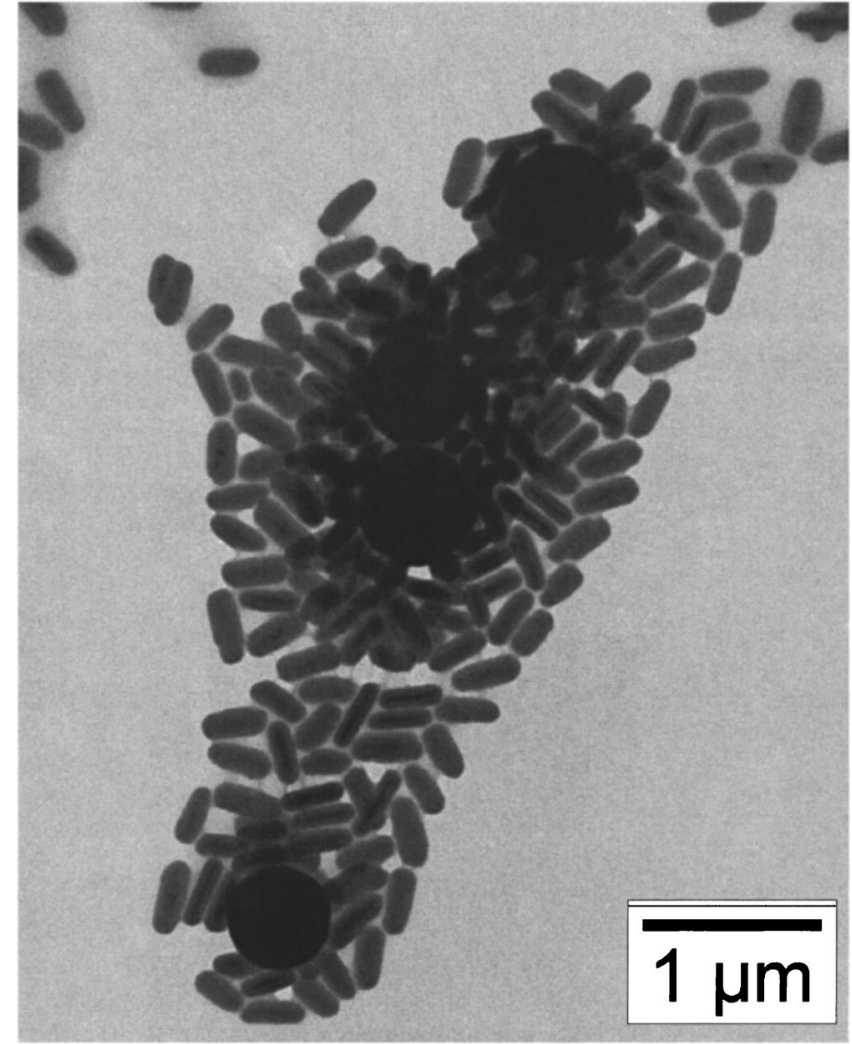

FIG. 4. Transmission electron microscope graph of a mixture of silica spheres and silica-coated goethite bars. The measures of the bare goethite nanorods are $L=230 \mathrm{~nm}, D=40 \mathrm{~nm}$, and $H=8 \mathrm{~nm}$, respectively. The particles have been grown with a $60 \mathrm{~nm}$ silica layer on each side.

The metastable fluid-fluid transition also shows this incorrect behavior for mixtures of spheres and plates ${ }^{35}$ and for bimodal mixtures of spheres. ${ }^{72}$ This behavior persists by including CS for the pressure of the spheres in the reservoir, which is strictly an inconsistency.

The fluid-solid binodal from SPT/PY, indicated by the dashed/dotted line in Fig. 5, is reminiscent of that from FMT/CS but gives a wider phase gap between fluid and solid. Similar observations have been made in the preceding section for mixtures of spheres and plates and can also be made for mixtures of bimodal spheres. This must be due to the fact that the PY compressibility equation of state always overestimates the true pressure. ${ }^{48}$ That is, at a given reservoir density a SPT/PY pressure and free-volume fraction is applied which is only found for higher reservoir volume fractions using FMT/CS.

\section{CONCLUSIONS}

We presented systematic and generic approaches to freevolume theory that is based on the characteristic measures of depletion agents, i.e., their volume, surface area, and integrate mean curvature, in a sea of spheres. This provides us with general "recipes" for calculating the phase behavior of mixtures of colloidal spheres with any dilute depletion agent.

First we derived the generalized free-volume theory from scaled-particle theory. Using an exact equation for the excluded volume, the free-volume fraction and pressure of the reservoir may be expressed in terms of the characteristic 

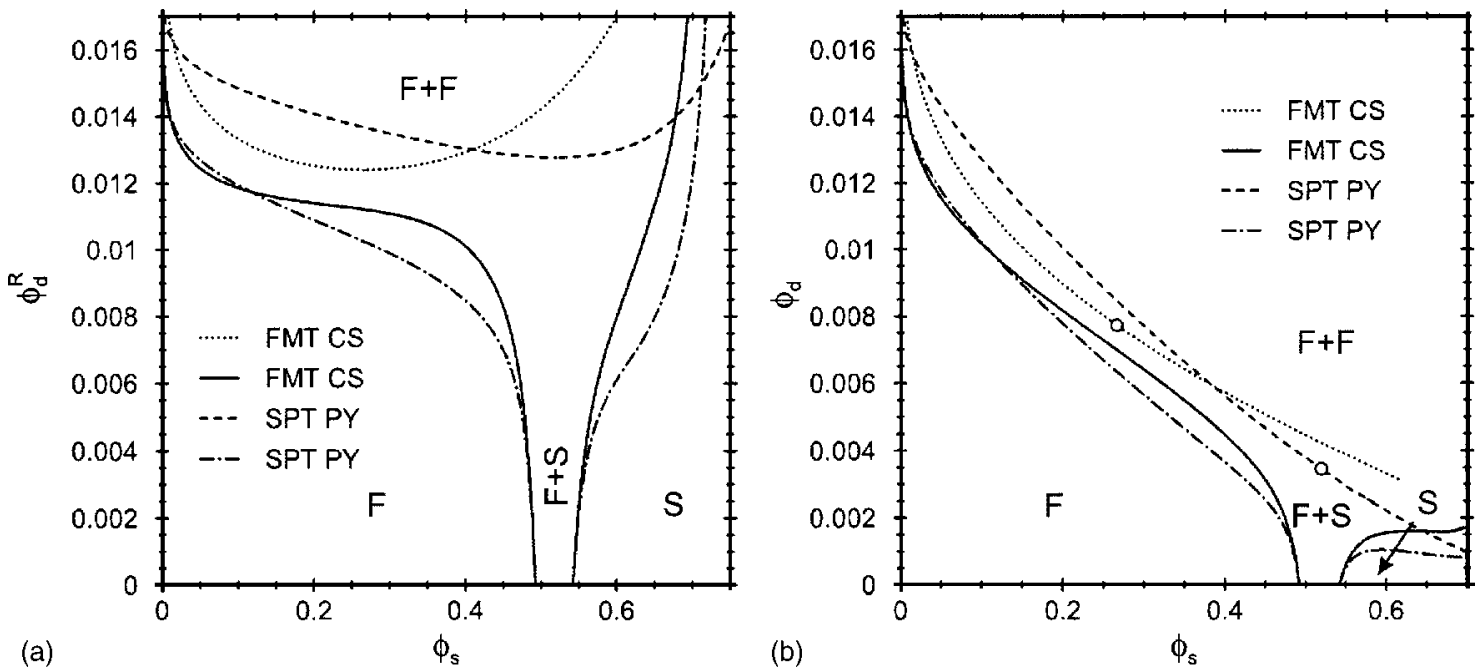

FIG. 5. The phase diagrams of a mixture of spheres and barlike mesogens from SPT, which maps to the PY compressibility equation, and FMT in the CS approach in both (a) the reservoir and (b) system representation. The measures are chosen to be such as found for goethite nanorods, $L=230 \mathrm{~nm}, D$ $=40 \mathrm{~nm}, H=8 \mathrm{~nm}$, and $\sigma=1000 \mathrm{~nm}$.

geometrical measures of the particles. These equations effectively amount to the Percus-Yevick compressibility equation of state, which is known to be a fair approximation only. This might cause the metastable fluid-fluid coexistence to become unphysical, whereas the fluid-solid coexistence may give too much broadening of the biphasic gap. This can slightly be improved by using the Carnahan-Starling equation of state for the spheres, but is strictly inconsistent with the approach. Moreover, general inclusion of a second-order expansion of all measures in the scaled-particle expression may lead to incorrect physics, as is the case for, e.g., spherocylinders, but is prerequisite for other shapes, e.g., cylinders.

Second, we argued that it is possible to recast the excess chemical potential of a depletion agent immersed in a sea of spheres into a sum over characteristic geometrical measures of the depletion agent times thermodynamic coefficients, as in Eq. (41). This approach is tested and numerically verified by extensive density functional theory calculation. ${ }^{54-56} \mathrm{We}$ have specified the thermodynamical coefficients for this fundamental-measure theory in Table II corresponding to the Percus-Yevick and Carnahan-Starling equation of state. The latter is most accurate and can be used thermodynamically consistent within the fundamental-measure approach. This makes the FMT/CS approach very attractive for future work besides its easier physical understanding and the fact that it yields similar or better results than SPT/PY.

We have shown three applications which indicate the potential of the present general approaches. For all our approaches it is also straightforward to generalize the freevolume theory to discrete and polydisperse mixtures. The combination of a polydisperse mixture of particles of different shape should be interesting in particular to experimental situations, when it is not always possible to prepare a monodisperse sample.

Finally, it is interesting to note that it is also possible to generalize for the shape of the colloidal species that are depleted. This mainly changes the thermodynamic potential, which would have to be rewritten in contributions only pro- portional to the characteristic measures, which are for three dimensions the particle's volume, area, mean curvature, and Euler characteristic.

\section{ACKNOWLEDGMENTS}

R.R. thanks Peter König, Klaus Mecke, Remco Tuinier, and Dirk Aarts for stimulating discussions. S.M.O. is deeply indebted to Rik Wensink and Henk Lekkerkerker for useful discussions and use of their notes and critically reading the manuscript. Matthias Schmidt is thanked for providing the basics in scaled-particle theory. Dominique Thies-Weesie, Henar Hernandez, and Chantal Vonk are gratefully acknowledged for providing the authors with the silica-coated goethite particles and the TEM picture of them with spheres. The work of SMO is part of the SoftLink research program of the "Stichting voor Fundamenteel Onderzoek der Materie (FOM)," which is financially supported by the "Nederlandse Organisatie voor Wetenschappelijk Onderzoek (NWO)."

${ }^{1}$ W. B. Russel, D. A. Saville, and W. R. Schowalter, Colloidal Dispersions (Cambridge University Press, Cambridge, 1989).

${ }^{2}$ D. F. Evans and H. Wennerström, The Colloidal Domain: Where Physics, Chemistry, Biology, and Technology Meet, 2nd ed. (Wiley-VCH, New York, 1999).

${ }^{3}$ S. Asakura and F. Oosawa, J. Chem. Phys. 22, 1255 (1954).

${ }^{4}$ S. Asakura and F. Oosawa, J. Polym. Sci. 32, 183 (1958).

${ }^{5}$ A. Vrij, Pure Appl. Chem. 48, 471 (1976).

${ }^{6}$ R. Roth, R. Evans, and S. Dietrich, Phys. Rev. E 62, 5360 (2000).

${ }^{7}$ S. Sanyal, N. Easwar, S. Ramaswamy, and A. K. Sood, Europhys. Lett. 18, 107 (1992).

${ }^{8}$ J. S. van Duijneveldt, A. W. Heinen, and H. N. W. Lekkerkerker, Europhys. Lett. 21, 369 (1993).

${ }^{9}$ P. D. Kaplan, J. L. Rouke, A. G. Yodh, and D. J. Pine, Phys. Rev. Lett. 72, 582 (1994).

${ }^{10}$ A. D. Dinsmore, A. G. Yodh, and D. J. Pine, Phys. Rev. E 52, 4045 (1995).

${ }^{11}$ U. Steiner, A. Meller, and J. Stavans, Phys. Rev. Lett. 74, 4750 (1995).

${ }^{12}$ A. Imhof and J. K. G. Dhont, Phys. Rev. Lett. 75, 1662 (1995).

${ }^{13}$ T. Biben and J. P. Hansen, Phys. Rev. Lett. 66, 2215 (1991).

${ }^{14}$ T. Biben and J. P. Hansen, J. Phys.: Condens. Matter 3, 65 (1991).

${ }^{15}$ H. N. W. Lekkerkerker and A. Stroobants, Physica A 195, 387 (1993).

${ }^{16}$ W. C. K. Poon and P. B. Warren, Europhys. Lett. 28, 513 (1994).

${ }^{17}$ Y. Rosenfeld, Phys. Rev. Lett. 72, 3831 (1994). 
${ }^{18}$ M. Dijkstra, R. van Roij, and R. Evans, Phys. Rev. Lett. 81, 2268 (1998).

${ }^{19}$ M. Dijkstra, R. van Roij, and R. Evans, Phys. Rev. Lett. 82, 117 (1999)

${ }^{20}$ M. Dijkstra, R. van Roij, and R. Evans, Phys. Rev. E 59, 5744 (1999).

${ }^{21}$ J. S. Rowlinson and B. Widom, Molecular Theory of Capillarity (Clarendon, Oxford, 1982) (Dover, reprint, 2002).

${ }^{22}$ R. Tuinier, J. Rieger, and C. G. de Kruif, Adv. Colloid Interface Sci. 103 1 (2003), and references therein.

${ }^{23}$ G. H. Koenderink, G. A. Vliegenthart, S. G. J. M. Kluijtmans, A. van Blaaderen, A. P. Philipse, and H. N. W. Lekkerkerker, Langmuir 15, 4683 (1999).

${ }^{24}$ G. A. Vliegenthart, A. van Blaaderen, and H. N. W. Lekkerkerker, Faraday Discuss. 112, 173 (1999).

${ }^{25}$ S. M. Oversteegen, J. E. G. J. Wijnhoven, C. Vonk, and H. N. W. Lekkerkerker, J. Phys. Chem. B 18, 18158 (2004).

${ }^{26}$ P. G. Bolhuis and D. Frenkel, J. Chem. Phys. 101, 9869 (1994).

${ }^{27}$ G. A. Vliegenthart and H. N. W. Lekkerkerker, J. Chem. Phys. 111, 4153 (1999).

${ }^{28}$ Z. Dogic, D. Frenkel, and S. Fraden, Phys. Rev. E 62, 3925 (2000).

${ }^{29}$ R. Roth, J. M. Brader, and M. Schmidt, Europhys. Lett. 63, 549 (2003).

${ }^{30}$ L. Helden, R. Roth, G. H. Koenderink, P. Leiderer, and C. Bechinger, Phys. Rev. Lett. 90, 048301 (2003).

${ }^{31}$ Y. Mao, M. E. Cates, and H. N. W. Lekkerkerker, Phys. Rev. Lett. 75, 4548 (1995).

${ }^{32}$ Y. Mao, M. E. Cates, and H. N. W. Lekkerkerker, J. Chem. Phys. 106, 3721 (1997).

${ }^{33}$ R. Roth, J. Phys.: Condens. Matter 15, S277 (2003).

${ }^{34}$ S. M. Oversteegen, C. Vonk, J. E. G. J. Wijnhoven, and H. N. W. Lekkerkerker, Phys. Rev. E 71, 041406 (2005).

${ }^{35}$ S. M. Oversteegen and H. N. W. Lekkerkerker, J. Chem. Phys. 120, 2470 (2004).

${ }^{36}$ L. Harnau and S. Dietrich, Phys. Rev. E 69, 051501 (2004).

${ }^{37}$ H. N. W. Lekkerkerker, W. C. K. Poon, P. N. Pusey, A. Stroobants, and P. B. Warren, Europhys. Lett. 20, 559 (1992).

${ }^{38}$ H. Reiss, H. L. Frisch, and J. L. Lebowitz, J. Chem. Phys. 31, 369 (1959).

${ }^{39}$ H. Reiss, H. L. Frisch, E. Helfand, and J. L. Lebowitz, J. Chem. Phys. 32 119 (1960)

${ }^{40}$ J. L. Lebowitz, E. Helfand, and E. Praestgaard, J. Chem. Phys. 43, 774 (1965).

${ }^{41}$ Y. Rosenfeld, J. Chem. Phys. 89, 4272 (1988).

${ }^{42}$ Y. Rosenfeld, Phys. Rev. Lett. 63, 980 (1989).

${ }^{43}$ Y. Rosenfeld, Phys. Rev. E 50, R3318 (1994).

${ }^{44}$ Y. Rosenfeld, Mol. Phys. 86, 637 (1995).
${ }^{45}$ M. Schmidt, H. Löwen, J. M. Brader, and R. Evans, Phys. Rev. Lett. 85, 1934 (2000).

${ }^{46}$ B. Widom, J. Chem. Phys. 39, 2808 (1963).

${ }^{47}$ N. F. Carnahan and K. E. Starling, J. Chem. Phys. 51, 635 (1969).

${ }^{48}$ J. P. Hansen and I. R. McDonald, Theory of Simple Liquids, 2nd ed. (Academic, New York, 1986).

${ }^{49}$ W. W. Wood, J. Chem. Phys. 20, 1334 (1952).

${ }^{50}$ D. Frenkel and A. J. C. Ladd, J. Chem. Phys. 81, 3188 (1984).

${ }^{51}$ J. Steiner, Mber. Preuss. Akad. Wiss. 2, 114 (1840); Gesammelte Werke (Chelsea Publ. Co., New York, 1971), Vol. 2.

${ }^{52}$ M. A. Cotter and D. C. Wacker, Phys. Rev. A 18, 2669 (1978).

${ }^{53}$ J. A. Barker and D. Henderson, Rev. Mod. Phys. 48, 587 (1976).

${ }^{54}$ P. Bryk, R. Roth, K. R. Mecke, and S. Dietrich, Phys. Rev. E 68, 031602 (2003).

${ }^{55}$ P.-M. König, R. Roth, and K. R. Mecke, Phys. Rev. Lett. 93, 160601 (2004).

${ }^{56}$ P.-M. König, P. Bryk, K. R. Mecke, and R. Roth, Europhys. Lett. 69, 832 (2005).

${ }^{57}$ G. A. Mansoori, N. F. Carnahan, K. E. Starling, and T. W. Leland, J. Chem. Phys. 54, 1523 (1971).

${ }^{58}$ R. Roth, R. Evans, A. Lang, and G. Kahl, J. Phys.: Condens. Matter 14, 12063 (2002).

${ }^{59}$ Y.-X Yu and J. Wu, J. Chem. Phys. 117, 10156 (2002).

${ }^{60}$ Y. Rosenfeld, J. Chem. Phys. 89, 4272 (1988).

${ }^{61}$ L. Onsager, Ann. N.Y. Acad. Sci. 51, 627 (1949).

${ }^{62}$ D. Frenkel, J. Chem. Phys. 91, 4912 (1987); 92, 5315(E) (1988).

${ }^{63}$ D. Frenkel and R. Eppenga, Phys. Rev. E 49, 1089 (1982).

${ }^{64}$ R. Eppenga and D. Frenkel, Mol. Phys. 52, 1303 (1984).

${ }^{65}$ S.-D. Zhang, P. A. Reynolds, and J. S. van Duijneveldt, J. Chem. Phys. 117, 9947 (2002).

${ }^{66}$ D. van der Beek, T. Schilling, and H. N. W. Lekkerkerker, J. Chem. Phys. 121, 5423 (2004).

${ }^{67}$ M. Piech and J. Y. Walz, J. Colloid Interface Sci. 232, 86 (2000).

${ }^{68}$ A. M. Wierenga, T. A. J. Lenstra, and A. P. Philipse, Colloids Surf., A 134, 359 (1998).

${ }^{69}$ B. J. Lemaire, P. Davidson, J. Ferré, J. P. Jamet, P. Panine, I. Dozov, and J. P. Jolivet, Phys. Rev. Lett. 88, 125507 (2002).

${ }^{70}$ B. J. Lemaire, P. Davidson, J. Ferré, J. P. Jamet, D. Petermann, I. Dozov, and J. P. Jolivet, Eur. Phys. J. E 13, 291 (2004).

${ }^{71}$ C. Graf, D. L. J. Vossen, A. Imhof, and A. van Blaaderen, Langmuir 19, 6693 (2003).

${ }^{72}$ J. M. Brader, Ph.D. thesis, University of Bristol, 2001. 